\title{
SMALL COMPOSITION OPERATORS ON ANALYTIC VECTOR-VALUED FUNCTION SPACES
}

\author{
Peide Liu, Eero Saksman and Hans-Olav Tylli
}

Let $\phi$ be an analytic mapping of the unit disk $D$ into itself. We characterize the weak compactness of the composition operator $C_{\phi}: f \mapsto f \circ \phi$ on the vector-valued Hardy space $H^{1}(X)$ $\left(=H^{1}(D, X)\right)$ and on the Bergman space $B_{1}(X)$, where $X$ is a Banach space. Reflexivity of $X$ is a necessary condition for the weak compactness of $C_{\phi}$ in each case. Assuming this, the operator $C_{\phi}: H^{1}(X) \rightarrow H^{1}(X)$ is weakly compact if and only if $\phi$ satisfies the Shapiro condition: $N_{\phi}(w)=o(1-|w|)$ as $|w| \rightarrow 1^{-}$, where $N_{\phi}$ stands for the Nevanlinna counting function of $\phi$. This extends a previous result of Sarason in the scalar case. Similarly, $C_{\phi}$ is weakly compact on $B_{1}(X)$ if and only if the angular derivative condition $\lim _{|w| \rightarrow 1^{-}}(1-|\phi(w)|) /(1-|w|)=\infty$ is satisfied. We also characterize the weak compactness of $C_{\phi}$ on vector-valued (little and big) Bloch spaces and on $H^{\infty}(X)$. Finally, we find conditions for weak conditional compactness of $C_{\phi}$ on the above mentioned spaces of analytic vector-valued functions.

\section{Introduction}

Let $\phi: D \rightarrow D$ be analytic, where $D$ is the open unit disk in C. Recently properties of the analytic composition operators

$$
f \mapsto f \circ \phi
$$

have been studied on the Hardy and Bergman spaces in numerous papers. Among the prominent results we mention the characterization [13] of compact composition operators on the Hardy space $H^{p}(1 \leq p<\infty)$ in terms of the Nevanlinna counting function $N_{\phi}$ of $\phi$ (see below for the definition of $N_{\phi}$ ). Thus, $C_{\phi}: H^{p} \rightarrow H^{p}$ is compact if and only if $N_{\phi}$ satisfies the Shapiro condition: $\quad N_{\phi}(w)=o(1-|w|)$ as $|w| \rightarrow 1^{-}$. Likewise, $C_{\phi}$ is compact on the Bergman space $B_{p}[\mathbf{9}]$ if and only if $\phi$ satisfies the angular derivative condition: $\lim _{|z| \rightarrow 1^{-}}(1-|\phi(z)|) /(1-|z|)=\infty$. Many other important properties of $C_{\phi}$ have also been characterized, such as spectral properties, membership in Schatten classes, closedness of the range and weak compactness. In addition, the same questions have been studied for composition operators acting 
on analytic functions defined on a domain in $\mathbf{C}^{n}$. We refer to the recent monographs [14] and [5] for an overview of the whole spectrum of present knowledge concerning analytic composition operators.

The purpose of this paper is to initiate the study of analytic composition operators on spaces of vector-valued analytic functions. Let $X$ be a Banach space and $1 \leq p<\infty$. The $X$-valued Hardy spaces $H^{p}(X)$ on the unit disk $D \subset \mathbf{C}$ are defined as follows

$$
\begin{aligned}
H^{p}(X)=\{f: D \rightarrow X \mid f \text { analytic }, & \|f\|_{H^{p}(X)}^{p} \\
: & \left.=\sup _{r<1} \frac{1}{2 \pi} \int_{0}^{2 \pi}\left\|f\left(r e^{i \theta}\right)\right\|_{X}^{p} d \theta<\infty\right\},
\end{aligned}
$$

for $p<\infty$ and in addition $\|f\|_{H^{\infty}(X)}=\sup _{z \in D}\|f(z)\|_{X}$. The natural definitions of the $X$-valued Bergman spaces $B_{p}(X)$ and Bloch spaces will be given later. Important parts of modern harmonic analysis have been extended to the vector-valued case during the last 15 years, and at the same time vector-valued harmonic analysis has had profound applications to classical harmonic analysis (see e.g. the reviews [4] and [11]). We continue this line of research by asking about basic properties of the vector-valued composition operators (1), where $f$ now belongs to some of the previously mentioned analytic vector-valued spaces.

We shall assume in the sequel that the Banach space $X$ is infinite-dimensional. In this case the operator $C_{\phi}$ is never compact. Namely, it fixes the constant functions (if $f(z) \equiv a \in X$, then $C_{\phi} f=f$ ) and hence a copy of $X$ itself. However, the question of weak compactness of $C_{\phi}$ remains equally interesting as an important 'smallness' property, and in this paper we shall concentrate on it.

A necessary condition for weak compactness of $C_{\phi}$ on $X$-valued spaces of analytic functions is the reflexivity of $X$. Assuming this, Theorem 3 below states that the operator $C_{\phi}$ is weakly compact on the Hardy space $H^{1}(X)$ if and only if $N_{\phi}$ satisfies the Shapiro condition and, moreover, $C_{\phi}: B_{1}(X) \rightarrow$ $B_{1}(X)$ is weakly compact if and only if $\phi$ satisfies the angular derivative condition. The first mentioned criterion extends the result of Sarason [12] stating that (in the scalar case) the weak compactness of $C_{\phi}: H^{1} \rightarrow H^{1}$ implies that $C_{\phi}$ is compact, i.e. the Shapiro condition holds. We refer to the remarks before Proposition 2 for the fact that the weak compactness of $C_{\phi}$ is obvious on $H^{p}(X)$ or $B_{p}(X)$ for $1<p<\infty$ and a reflexive space $X$.

Theorem 4 characterizes weak compactness of $C_{\phi}$ on the vector-valued (big and little) Bloch-spaces $\mathcal{B}(X)$ and $\mathcal{B}_{0}(X)$. This generalizes and extends [10, Theorems 1 and 3], where scalar composition operators on the Bloch spaces were studied. As a special case of Theorem 4 we obtain that 
[10, Theorem 3] also holds for the big Bloch space $\mathcal{B}$ : Every weakly compact composition operator on $\mathcal{B}$ is compact. Theorem 6 settles the case $H^{\infty}(X)$.

Finally, Theorem 7 characterizes weak conditional compactness of $C_{\phi}$ on (most of) the vector-valued spaces of analytic functions mentioned above. The conditions for this turn out to be similar to those for weak compactness, the difference being that instead of the reflexivity of $X$ one has to assume that $\ell^{1}$ does not embed into $X$.

\section{Results.}

Throughout this paper $\phi$ stands for an analytic self-map of $D$ and $X$ is a Banach space. We first recall the definitions of weakly compact operators and the standard vector-valued analytic spaces that we shall consider. If $E$ is a Banach space the bounded linear operator $T: E \rightarrow E$ is weakly compact if the image $T B_{E}$ of the unit ball $B_{E}$ is relatively compact in the weak topology of $E$. Another way to state this is to demand that every bounded sequence $\left(x_{k}\right) \subset E$ has a subsequence $\left(x_{k_{i}}\right)$ such that the sequence $\left(T x_{k_{i}}\right)$ converges weakly (the equivalence of the stated conditions follows from the Eberlein-Smulian theorem [18, II.c.3 ]).

The vector-valued Hardy spaces have already been defined in (2). The definition of the $X$-valued Bergman spaces is analogous:

$$
B_{p}(X)=\left\{f: D \rightarrow X \mid f \text { analytic, }\|f\|_{B_{p}(X)}^{p}:=\frac{1}{\pi} \int_{D}\|f(z)\|_{X}^{p} d A(z)<\infty\right\},
$$

where $d A$ denotes the two-dimensional Lebesgue measure. The Bloch space $\mathcal{B}(X)$ is defined in a natural manner:

$$
\begin{aligned}
& \mathcal{B}(X)=\left\{f: D \rightarrow X \mid f \text { analytic, }\|f\|_{\mathcal{B}(X)}\right. \\
&\left.:=\|f(0)\|_{X}+\sup _{z \in D}\left(1-|z|^{2}\right)\left\|f^{\prime}(z)\right\|_{X}<\infty\right\} .
\end{aligned}
$$

The little Bloch space $\mathcal{B}_{0}(X)$ is the closed subspace of $\mathcal{B}(X)$ consisting of the analytic functions $f$ with the property $\lim _{|z| \rightarrow 1}\left(1-|z|^{2}\right)\left\|f^{\prime}(z)\right\|_{X}=0$. The completeness of these spaces is established exactly as in the scalar case. We shall write, e.g., $H^{1}=H^{1}(\mathbf{C})$.

Consider next the boundedness of the operator $C_{\phi}$ on these vector-valued spaces.

It is well known that for analytic $f: D \rightarrow X$ the norm $z \mapsto\|f(z)\|_{X}$ is subharmonic. Namely, it may be expressed as the (continuous) supremum of a family of subharmonic functions:

$$
\|f(z)\|_{X}=\sup _{x^{\prime} \in B_{X^{\prime}}}\left|\left\langle x^{\prime}, f(z)\right\rangle\right|,
$$


where $B_{X^{\prime}}$ stands for the unit ball of the dual space $X^{\prime}$. Hence we may apply the Littlewood subordination theorem in the well known manner (see [5, Theorem 3.1]) to deduce that $C_{\phi}$ is contractive on the spaces $H^{p}(X)$ and $B_{p}(X)(p \in[1, \infty[)$ in the case that $\phi(0)=0$. In order to treat the general case we note that it is enough to verify that $C_{\phi}$ is bounded whenever $\phi$ is a conformal automorphism of the disk onto itself. In this case boundedness is established by the simple change of variables $\phi(z)=w$ inside the integral defining the norm of $C_{\phi} f$. However, in the case of Hardy spaces one first applies the change of variables $\phi(z)=w$ to $C_{\phi}(f(r z))$ and then lets $r$ increase to 1 , using the subharmonicity of the norm $\|f(z)\|_{X}$. The reason for the extra care is the fact that, contrary to the scalar case, the functions in $H^{p}(X)$ need not in general have radial boundary values a.e.. In fact, elements in $H^{p}(X)$ can be identified with $X$-valued $L_{p}$-functions on the boundary with vanishing negative Fourier coefficients if and only if $X$ has the so called analytic Radon-Nikodým property (see for instance [2]). The boundedness of $C_{\phi}$ on the Bloch spaces reduces to the scalar case treated in [10] by noting that $\|f\|_{\mathcal{B}(X)} \leq 2 \sup _{\lambda \in B_{X}}\|\lambda \circ f\|_{\mathcal{B}}$. Summing up, we have established (the non-trivial parts) of the following:

Proposition 1. Let $X$ be a Banach space and $\phi: D \rightarrow D$ be analytic. The operator $C_{\phi}$ is bounded on the spaces $\mathcal{B}(X), H^{p}(X)$ and $B_{p}(X)$ for $1 \leq p \leq \infty$. Moreover, $C_{\phi}$ maps $\mathcal{B}_{0}(X)$ into itself only if $\phi \in \mathcal{B}_{0}$. In the case where $\phi(0)=0$ the operator $C_{\phi}$ is actually a contraction on the Hardy and Bergman spaces.

From here on we may and shall always assume in the proofs of our theorems that $\phi(0)=0$, since neither the results nor the conditions on $\phi$ will change if $\phi$ is composed with a conformal self-map of $D$.

We start by considering composition operators on $X$-valued Bergman and Hardy spaces. Assuming that $C_{\phi}$ is weakly compact on $B_{p}(X)$ or on $H^{p}(X)$ we note that $X$ is reflexive. Namely, because $C_{\phi}$ fixes constant functions we see that $C_{\phi}$ fixes a copy of the space $X$ itself. Hence, for $p \in(1, \infty)$ the question of weak compactness of $C_{\phi}$ is trivial since the spaces $B_{p}(X)$ and $H^{p}(X)$ are reflexive as closed subspaces of the reflexive Banach spaces $L^{p}(D, X)$ and $L^{p}(\partial D, X)$. For these facts, see [6, Corollary IV.2] and note that reflexive Banach spaces possess the Radon-Nikodým property [6, Corollary III.13] and consequently also the analytic Radon-Nikodým property (cf. [2]).

The following simple proposition is based on well-known properties of the de la Vallée-Poussin summability kernels.

Proposition 2. Let $X$ be a Banach space and let $n \in \mathbf{N}$. Define the 
operator $V_{n}$ by setting

$$
V_{n} f(z)=\sum_{k=0}^{n} \hat{f}_{k} z^{k}+\sum_{k=n+1}^{2 n-1} \frac{2 n-k}{n} \hat{f}_{k} z^{k}
$$

for analytic $f: D \rightarrow X$ with the Fourier expansion $f=\sum_{k=0}^{\infty} \hat{f}_{k} z^{k}$. Then

$$
\left\|V_{n} f\right\|_{H^{1}(X)} \leq 2\|f\|_{H^{1}(X)}
$$

for all $f \in H^{1}(X)$. In addition, given $\varepsilon>0$ and $r \in(0,1)$ there is $n_{0}=$ $n_{0}(\varepsilon, r)>0$ such that

$$
\left\|f(z)-V_{n_{0}} f(z)\right\|_{X} \leq \varepsilon\|f\|_{H^{1}(X)} \quad \text { for all }|z| \leq r \text { and } f \in H^{1}(X) .
$$

Moreover, if $X$ is reflexive, then the operator $V_{n}: H^{1}(X) \rightarrow H^{1}(X)$ is weakly compact for all $n \geq 1$. All the statements hold also if $H^{1}(X)$ is replaced by one of the spaces $H^{\infty}(X)$ and $B_{1}(X)$.

Proof. We commence with the Hardy space $H^{1}(X)$. Let $f \in H^{1}(X)$ and assume first that $f$ is continuous up to the boundary $\partial D$. Then $V_{n}$ (viewed as operating on boundary values) equals convolution with the de la ValleePoussin kernel so that $V_{n} f=\left(2 K_{2 n-1}-K_{n-1}\right) * f$, where $K_{n}$ denotes the Fejer kernel. Claim (4) follows since $K_{n}$ is a summability kernel (see [7], p. 12). In order to prove (5), let $r \in(0,1)$ and $\varepsilon>0$. The Poisson integral representation yields the estimate $\|f(z)\|_{X} \leq C(1-|z|)^{-1}\|f\|_{H^{1}(X)}$, with $C$ independent of $f$. Choose $n_{0}$ large enough so that $3 C r^{n_{0}}(1-r)^{-1} \leq \varepsilon$. Given $f \in H^{1}(X)$, write $f-V_{n_{0}} f=z^{n_{0}} g$ with $\|g\|_{H^{1}(X)}=\left\|f-V_{n_{0}} f\right\|_{H^{1}(X)}$ and deduce that

$$
\begin{aligned}
\left\|\left(\mathrm{I}-V_{n_{0}}\right) f(z)\right\|_{X} & =|z|^{n_{0}}\|g(z)\|_{X} \leq C r^{n_{0}}(1-r)^{-1}\left\|f-V_{n_{0}} f\right\|_{H^{1}(X)} \\
& \leq(\varepsilon / 3)\left\|f-V_{n_{0}} f\right\|_{H^{1}(X)},
\end{aligned}
$$

for $|z| \leq r$. This yields (5) in view of (4). In the case that $f$ is not continuous we denote $f_{s}(z)=f(s z)$ for $0<s<1$ and observe that $V_{n} f_{s}=\left(V_{n} f\right)_{s}$. Hence we get, e.g., that $\left\|\left(V_{n} f\right)_{s}\right\|_{H^{1}(X)} \leq 2\left\|f_{s}\right\|_{H^{1}(X)}$ and by letting $s$ increase to 1 we obtain (4) and (5) for $f$. The proof for $H^{\infty}(X)$ is entirely similar.

We next assume that $f \in B_{1}(X)$. The uniform boundedness of the operators $V_{n}$ follows easily by writing

$$
\|f\|_{B_{1}(X)}=2 \int_{0}^{1}\left\|f_{r}\right\|_{H^{1}(X)} r d r
$$

and applying the $H^{1}(X)$-boundedness together with the relation $V_{n} f_{r}=$ $\left(V_{n} f\right)_{r}$. Towards $(5)$, suppose that $r \in(1 / 2,1)$ and $\varepsilon>0$ are given and 
that $f \in B_{1}(X)$ with $\|f\|_{B_{1}(X)} \leq 1$. Set $s=\sqrt{r}$. Since (5) holds for $H^{1}(X)$ we may choose $n_{0}$ such that $\left\|g(z)-V_{n_{0}} g(z)\right\|_{X} \leq \frac{1}{2} \varepsilon(1-s)\|g\|_{H^{1}(X)}$ for $|z| \leq s$ and all $g \in H^{1}(X)$. The subharmonicity of $z \mapsto\|f(z)\|_{X}$ yields that $\left\|f_{r}\right\|_{H^{1}(X)}$ increases with $r$, so that (6) yields a radius $r^{\prime} \in(s, 1)$ with $\left\|f_{r^{\prime}}\right\|_{H^{1}(X)} \leq 2(1-s)^{-1}$. Hence, for $|z| \leq r$ we have that $\left|z / r^{\prime}\right| \leq|z / s| \leq s$ and we may apply our assumption on $n_{0}$ to the function $f_{r^{\prime}}$ and deduce that

$\left\|f(z)-V_{n_{0}} f(z)\right\|_{X}=\left\|f_{r^{\prime}}\left(z / r^{\prime}\right)-V_{n_{0}} f_{r^{\prime}}\left(z / r^{\prime}\right)\right\|_{X} \leq \frac{1}{2} \varepsilon(1-s)\left\|f_{r^{\prime}}\right\|_{H^{1}(X)} \leq \varepsilon$.

Finally, assume that $X$ is reflexive. We fix $n$ and denote $G_{n}=\left(\bigoplus_{0}^{2 n-1} X\right)_{\ell^{2}}$. For any analytic function $f: D \rightarrow X$ with Fourier coefficients $\hat{f}(k), k \geq 0$, define $S_{n} f=(\hat{f}(0), \hat{f}(1), \ldots, \hat{f}(2 n-1)) \in G_{n}$ and for any $x=\left(x_{0}, \ldots\right.$, $\left.x_{2 n-1}\right) \in G_{n}$ set

$$
\left(R_{n} x\right)(z)=\left(x_{0}+x_{1} z+\ldots x_{n} z^{n}+x_{n+1} \frac{n-1}{n} z^{n+1}+\ldots+x_{2 n-1} \frac{1}{n} z^{2 n-1}\right)
$$

for $z \in D$. It is clear that the operators $S_{n}: Y \rightarrow G_{n}$ and $R_{n}: G_{n} \rightarrow Y$ are well-defined and bounded if $Y$ is any of the spaces $H^{1}(X), H^{\infty}(X)$ or $B_{1}(X)$. Hence the operator $V_{n}=R_{n} S_{n}$ is weakly compact since it factorizes through the reflexive space $G_{n}$ (cf. [18, II.C.5]).

We recall the Stanton formula [16, Theorem 2] for continuous subharmonic functions $u: D \rightarrow \mathbf{R}$,

$$
\frac{1}{2 \pi} \int_{0}^{2 \pi} u\left(\phi\left(r e^{i \theta}\right)\right) d \theta=u(0)+\frac{1}{2 \pi} \int_{D} N_{\phi}(r, w) d[\Delta u](w),
$$

where $r \in(0,1)$ and the standing assumption $\phi(0)=0$ has been taken into account. Above $d[\Delta u](w)$ denotes integration with respect to the distributional Laplacian of $u$, which is a positive measure on $D$ since $u$ is subharmonic. In addition, $N_{\phi}(r, \cdot)$ denotes the partial Nevanlinna counting function of $\phi$ :

$$
N_{\phi}(r, w)=\sum_{z \in \phi^{-1}(w),|z| \leq r} \log \left(\frac{r}{|z|}\right)
$$

for $r \in(0,1)$. The (standard) Nevanlinna counting function $N_{\phi}$ is defined as $N_{\phi}(w)=N_{\phi}(1, w)=\sum_{z \in \phi^{-1}(w)} \log (1 /|z|)$.

Let $f: D \rightarrow X$ be an analytic map. By applying the Stanton formula to the subharmonic function $z \mapsto\|f(z)\|_{X}$ we obtain

(9) $\frac{1}{2 \pi} \int_{0}^{2 \pi}\left\|f\left(\phi\left(r e^{i \theta}\right)\right)\right\|_{X} d \theta=\|f(0)\|_{X}+\frac{1}{2 \pi} \int_{D} N_{\phi}(r, w) d\left[\Delta\left(\|f\|_{X}\right)\right](w)$. 
Since $N_{\phi}(r, w)$ increases monotonically to $N_{\phi}(w)$ as $r$ increases to 1 , an application of the monotone convergence theorem yields

$$
\left\|C_{\phi} f\right\|_{H^{1}(X)}=\|f(0)\|_{X}+\frac{1}{2 \pi} \int_{D} N_{\phi}(w) d\left[\Delta\left(\|f\|_{X}\right)\right](w) .
$$

This formula can be viewed as a generalization of the well-known LittlewoodPaley identity. The technique of applying the Stanton formula for the Hardy spaces $H^{p}$ with $p \neq 2$ was introduced by Shapiro and Sundberg [15], where (10) appears in the scalar case.

We proceed to derive the corresponding formula for the Bergman norm. Note first that $2 \int_{|z|}^{1} r \log (r /|z|) d r=B(|z|)$, where $B(u)=\log (1 / u)-(1-$ $\left.u^{2}\right) / 2$ for $u \in(0,1)$. Combining this observation with (8) we easily see that

$$
\int_{0}^{1} r N_{\phi}(r, w) d r=\sum_{z \in \phi^{-1}(w)} B(|z|)=: N_{\phi}^{B}(w) .
$$

Multiply (9) by $2 r$ and integrate from 0 to 1 with respect to $r$ in order to obtain the exact formula

$$
\left\|C_{\phi} f\right\|_{B_{1}(X)}=\|f(0)\|_{X}+\frac{1}{2 \pi} \int_{D} N_{\phi}^{B}(w) d\left[\Delta\left(\|f\|_{X}\right)\right](w) .
$$

However, we introduce an equivalent norm that is easier to handle. Note first that $B(1-s)=s^{2}+s^{3} / 3+s^{4} / 4+\ldots$ and $-s \log (1-s)=s^{2}+s^{3} / 2+s^{4} / 3+\ldots$ for $s \in(0,1)$. Consequently $(3 / 4)(1-u) \log (1 / u) \leq B(u) \leq(1-u) \log (1 / u)$. Thus, by introducing a modified Nevanlinna counting function

$$
\widetilde{N}_{\phi}(w)=\sum_{z \in \phi^{-1}(w)}(1-|z|) \log (1 /|z|)
$$

we arrive at an equivalent expression for the Bergman norm of $C_{\phi} f$ :

$$
\left\|C_{\phi} f\right\|_{B_{1}(X)} \sim\|f(0)\|_{X}+\frac{1}{2 \pi} \int_{D} \tilde{N}_{\phi}(w) d\left[\Delta\left(\|f\|_{X}\right)\right](w) .
$$

Important special cases of the previous formulas are obtained if we choose $\phi$ to be the identity map. In the first place

$$
\|f\|_{H^{1}(X)}=\|f(0)\|_{X}+\frac{1}{2 \pi} \int_{D} \log \left(\frac{1}{|w|}\right) d\left[\Delta\left(\|f\|_{X}\right)\right](w),
$$

and for the Bergman norm there is the equivalence

$$
\|f\|_{B_{1}(X)} \sim\|f(0)\|_{X}+\frac{1}{2 \pi} \int_{D}(1-|w|) \log \left(\frac{1}{|w|}\right) d\left[\Delta\left(\|f\|_{X}\right)\right](w) .
$$


The following result extends and complements the scalar results of MacCluer and Shapiro [9], Shapiro [13] and Sarason [12].

\section{Theorem 3.}

(i) $C_{\phi}: B_{1}(X) \rightarrow B_{1}(X)$ is weakly compact if and only if $X$ is reflexive and

$$
\lim _{|w| \rightarrow 1^{-}}(1-|\phi(w)|) /(1-|w|)=\infty .
$$

(ii) $C_{\phi}: H^{1}(X) \rightarrow H^{1}(X)$ is weakly compact if and only if $X$ is reflexive and

$$
N_{\phi}(w)=o(1-|w|) \text { as }|w| \rightarrow 1^{-} .
$$

Proof. (i) If $C_{\phi}: B_{1}(X) \rightarrow B_{1}(X)$ is weakly compact we have already seen that $X$ must be reflexive. Fix a vector $x_{0} \in X$ with $\left\|x_{0}\right\|_{X}=1$ and consider the subspace $M \subset B_{1}(X)$, where $M=\left\{x_{0} f(z) \mid f(z) \in B_{1}\right\}$. Clearly $C_{\phi}: M \rightarrow M$ and the map $C_{\phi}: M \rightarrow M$ is 'isomorphic' to the scalar composition operator $C_{\phi}: B_{1} \rightarrow B_{1}$. Hence the scalar operator $C_{\phi}: B_{1} \rightarrow B_{1}$ is weakly compact. Now $B_{1}$ is isomorphic to $\ell^{1}$ (see [18, III.A.11]) and every weakly compact operator on $\ell^{1}$ is actually compact, since $\ell^{1}$ has the Schur property [18, III.C.9]. Hence $C_{\phi}: B_{1} \rightarrow B_{1}$ is compact and this forces $\phi$ to satisfy the angular derivative criterion (16) according to [9, Theorem 3.5].

In order to prove the other direction, we modify ideas due to Shapiro and Sundberg [15]. We assume the conditions of part (i) and consider the operators $T_{n}$ :

$$
T_{n}=C_{\phi}-C_{\phi} V_{n}: B_{1}(X) \rightarrow B_{1}(X),
$$

where $n \geq 1$ and $V_{n}$ is the weakly compact operator provided by Proposition 2. It is enough to establish that the operator norm of $T_{n}$ tends to zero as $n \rightarrow \infty$, since the weakly compact operators form a closed ideal of $L\left(B_{1}(X)\right)$ (see [18, II.c.6]). Suppose thus that $\varepsilon>0$ is given. Condition (16) yields $r \in(1 / 2,1)$ so that $1-|w| \leq \varepsilon(1-|\phi(w)|)$ for $|w| \in(r, 1)$. For these values of $w$ we may combine the Littlewood inequality $N_{\phi}(w) \leq \log (1 /|w|$ ) (see [14, Corollary 10.4(b)]) with definition (12) and deduce that

$$
\widetilde{N}_{\phi}(w) \leq \varepsilon(1-|w|) N_{\phi}(w) \leq \varepsilon(1-|w|) \log (1 /|w|)
$$

for $|w| \geq r$. We next fix a cut-off function $\psi$ that will be needed later in the argument: Let $\psi \in C_{0}^{\infty}(D)$ satisfy $0 \leq \psi(z) \leq 1, \psi(z)=1$ for $|z| \leq r$ and $\psi(z)=0$ for $|z| \geq(1+r) / 2$. Proposition 2 yields $n \in \mathbf{N}$ such that

$$
\left\|f(z)-V_{n} f(z)\right\|_{X} \leq \min \left\{\varepsilon, 1 / \sup _{w \in D}|\Delta \psi(w)|\right\}\|f\|_{B_{1}(X)}
$$


for $z \in \operatorname{supp}(\psi)$ and all $f \in B_{1}(X)$.

Assume next that $f \in B_{1}(X)$ is arbitrary. As $\left(\mathrm{I}-V_{n}\right) f(0)=0$ we may invoke formula (13) in the form

$$
\begin{aligned}
\left\|T_{n} f\right\|_{B_{1}(X)} \sim & \frac{1}{2 \pi} \int_{r<|w|<1} \tilde{N}_{\phi}(w) d\left[\Delta\left(\left\|\left(\mathrm{I}-V_{n}\right) f\right\|_{X}\right)\right](w) \\
& +\frac{1}{2 \pi} \int_{|w| \leq r} \tilde{N}_{\phi}(w) d\left[\Delta\left(\left\|\left(\mathrm{I}-V_{n}\right) f\right\|_{X}\right)\right](w)=: A+B,
\end{aligned}
$$

say. The first term is easily estimated using (18) and (15):

$$
\begin{aligned}
A & \leq \frac{1}{2 \pi} \int_{r<|w|<1} \varepsilon(1-|w|) \log \left(\frac{1}{|w|}\right) d\left[\Delta\left(\left\|\left(\mathrm{I}-V_{n}\right) f\right\|_{X}\right)\right](w) \\
& \leq 2 \varepsilon\left\|\left(\mathrm{I}-V_{n}\right) f\right\|_{B_{1}(X)} \leq 6 \varepsilon\|f\|_{B_{1}(X)}
\end{aligned}
$$

where the extra constant 2 comes from the fact that we used the equivalent norm (15). In order to bound the second term we use the crude estimate $\widetilde{N}_{\phi}(w) \leq \log (1 /|w|)$, which follows from (12) and the Littlewood inequality, and apply the substitution $w=r w^{\prime}$ :

$$
\begin{aligned}
B \leq & \frac{1}{2 \pi} \int_{|w| \leq r} \log \left(\frac{1}{|w|}\right) d\left[\Delta\left(\left\|\left(\mathrm{I}-V_{n}\right) f\right\|_{X}\right)\right](w) \\
\leq & \frac{1}{2 \pi} \int_{D} \log \left(1 /\left|w^{\prime}\right|\right) d\left[\Delta\left(\left\|\left(\mathrm{I}-V_{n}\right) f_{r}\right\|_{X}\right)\right]\left(w^{\prime}\right)+ \\
& \quad+\frac{1}{2 \pi} r^{2} \log \left(\frac{1}{r}\right) \int_{D} d\left[\Delta\left(\left\|\left(\mathrm{I}-V_{n}\right) f_{r}\right\|_{X}\right)\right]\left(w^{\prime}\right)=: E+F .
\end{aligned}
$$

According to (14) and (19) we obtain that $E=\left\|\left(\mathrm{I}-V_{n}\right) f_{r}\right\|_{H^{1}(X)} \leq$ $2 \varepsilon\|f\|_{B_{1}(X)}$. The remaining term can be taken care of as follows:

$$
\begin{aligned}
F & =\frac{1}{2 \pi} \log \left(\frac{1}{r}\right) \int_{r D} d\left[\Delta\left(\left\|\left(\mathrm{I}-V_{n}\right) f\right\|_{X}\right)\right](w) \\
& \leq \frac{1}{2 \pi} \log (1 / r) \int_{D} \psi(w) d\left[\Delta\left(\left\|\left(\mathrm{I}-V_{n}\right) f\right\|_{X}\right)\right](w) \\
& =\frac{1}{2 \pi} \log (1 / r) \int_{D} \Delta \psi(w)\left\|\left(\mathrm{I}-V_{n}\right) f(w)\right\|_{X} d w \leq 2 \varepsilon\|f\|_{B_{1}(X)},
\end{aligned}
$$

according to (19). We deduce that $\left\|T_{n} f\right\|_{B_{1}(X)} \leq C \varepsilon\|f\|_{B_{1}(X)}$ with $C$ independent of $\varepsilon$ and $f$, which finishes the proof.

(ii) For the necessity of the stated conditions we argue as above in part (i). Here we apply the result of Sarason [12] stating that weakly compact (scalar) composition operators $C_{\phi}: H^{1} \rightarrow H^{1}$ are compact. Condition (17) follows when this is combined with the Shapiro characterization [13, Theorem 2.3] of compact composition operators on $H^{2}$ and the 
well-known fact that compactness of $C_{\phi}$ on $H^{p}$ does not depend on $p$ (cf. [5, Theorem 3.12]).

The proof of the sufficiency follows the same outline as the one of part (i), albeit being slightly simpler, as it now suffices to use the standard Nevanlinna counting function. Hence we omit the details.

Recall that the Banach space $E$ has the Dunford-Pettis property if for every pair of weakly null sequences $\left(x_{n}^{\prime}\right) \in E^{\prime}$ and $\left(x_{n}\right) \in E$ we have $\lim _{n \rightarrow \infty}\left\langle x_{n}^{\prime}, x_{n}\right\rangle=0$. As a consequence, it follows that if $T: E \rightarrow E$ and $S: E \rightarrow E$ are weakly compact linear operators, then $T S$ is compact. Moreover, $T$ maps weakly null sequences to norm null sequences. We refer to [18, III.D.33-34] for these facts. We will apply this useful concept when dealing with vector-valued Bloch spaces in the proof of the following theorem, which extends [10, Theorem 3 (see also Theorems 1 and 2)].

\section{Theorem 4.}

(i) $C_{\phi}: \mathcal{B}(X) \rightarrow \mathcal{B}(X)$ is weakly compact if and only if $X$ is reflexive and

$$
\lim _{a \rightarrow 1^{-}} \sup _{\{z \in D:|\phi(z)| \geq a\}} \frac{\left(1-|z|^{2}\right)\left|\phi^{\prime}(z)\right|}{1-|\phi(z)|^{2}}=0 .
$$

(ii) $C_{\phi}: \mathcal{B}_{0}(X) \rightarrow \mathcal{B}_{0}(X)$ is weakly compact if and only if $X$ is reflexive and

$$
\lim _{|z| \rightarrow 1^{-}} \frac{\left(1-|z|^{2}\right)\left|\phi^{\prime}(z)\right|}{1-|\phi(z)|^{2}}=0
$$

Proof. (i) We first prove the necessity. Exactly as in the proof of Theorem 3.(i) it suffices to show that condition (20) follows if the scalar operator $C_{\phi}: \mathcal{B} \rightarrow \mathcal{B}$ is weakly compact. For this purpose we produce a weakly compact counterpart of the necessity argument of [10, Theorem 2]. Assume thus that $C_{\phi}: \mathcal{B} \rightarrow \mathcal{B}$ is weakly compact, but (20) does not hold. Then there is a sequence $\left(z_{n}\right)$ in $D$ and a constant $\varepsilon>0$ so that $\left|\phi\left(z_{n}\right)\right| \rightarrow 1$ as $n \rightarrow \infty$ together with

$$
\frac{\left(1-\left|z_{n}\right|^{2}\right)\left|\phi^{\prime}\left(z_{n}\right)\right|}{1-\left|\phi\left(z_{n}\right)\right|^{2}} \geq \varepsilon \quad \text { for each } n \geq 1
$$

Denote $w_{n}=\phi\left(z_{n}\right)$. By multiplying $\phi$ by a unimodular constant and extracting a subsequence, if needed, we may also assume that $w_{n} \rightarrow 1$ as $n \rightarrow \infty$ and $\left|\left(w_{2 n}-w_{2 n+1}\right) /\left(1-\bar{w}_{2 n} w_{2 n+1}\right)\right| \geq 1 / 2$ for all $n \geq 1$. Define

$$
f_{n}(z)=\log \left(\frac{1-\bar{w}_{2 n} z}{1-\bar{w}_{2 n+1} z}\right)
$$


for $n \geq 1$ and $z \in D$. Clearly $f_{n} \in \mathcal{B}_{0}$ and a simple computation shows that $\left\|f_{n}\right\|_{\mathcal{B}_{0}} \leq 4$ for each $n \geq 1$. Observe that for each $k \geq 1$ it holds that $\hat{f}_{n}(k) \rightarrow 0$ as $n \rightarrow \infty$. Hence, if $g$ is a polynomial one obtains

$$
\lim _{n \rightarrow \infty}\left\langle g, f_{n}\right\rangle=\lim _{n \rightarrow \infty} \int_{D} \overline{g(z)} f_{n}(z) d A(z)=0,
$$

where the brackets refer to the duality $\mathcal{B}_{0}{ }^{\prime}=B_{1}$ (see [19, Theorem 5.2.8]). Since polynomials are dense in $B_{1}$ and the sequence $\left(f_{n}\right)$ is bounded we conclude that $f_{n} \rightarrow 0$ weakly in $\mathcal{B}_{0}$, and hence in $\mathcal{B}$. However, $\mathcal{B}_{0}$ certainly has the Dunford-Pettis property as $\mathcal{B}_{0}{ }^{\prime}=B_{1} \approx \ell_{1}$ has the Schur property. This fact and the weak compactness of $C_{\phi}$ yield that the sequence $\left(C_{\phi} f_{n}\right)$ is norm null in $\mathcal{B}$. But this is impossible since using (22) we may estimate that for each $n \geq 1$

$$
\begin{aligned}
\left\|C_{\phi} f_{n}\right\|_{\mathcal{B}} & \geq\left(1-\left|z_{2 n}\right|^{2}\right)\left|\left(f_{n} \circ \phi\right)^{\prime}\left(z_{2 n}\right)\right| \\
& \geq \varepsilon\left(1-\left|w_{2 n}\right|^{2}\right)\left|\frac{-\bar{w}_{2 n}}{1-\left|w_{2 n}\right|^{2}}+\frac{\bar{w}_{2 n+1}}{1-\bar{w}_{2 n+1} w_{2 n}}\right| \\
& =\varepsilon\left|\left(\bar{w}_{2 n}-\bar{w}_{2 n+1}\right) /\left(1-w_{2 n} \bar{w}_{2 n+1}\right)\right| \geq \varepsilon / 2,
\end{aligned}
$$

and we are done.

Towards the converse direction, assume that $X$ is reflexive and (20) holds. Define the operator $\widetilde{V}_{n}$ by setting $\widetilde{V}_{n} f(z)=f(0)+\int_{0}^{z} V_{n} f^{\prime}(w) d w$, where $V_{n}$ is the operator provided by Proposition 1 . Clearly $\widetilde{V}_{n} f$ is analytic and by applying Proposition 1 and the maximum principle we see that

$$
\begin{aligned}
\sup _{|z|=r}\left\|\left(\widetilde{V}_{n} f\right)^{\prime}(z)\right\|_{X} & =\sup _{|z|=r}\left\|V_{n} f^{\prime}(z)\right\|_{X}=\sup _{|w|=1}\left\|V_{n} f_{r}^{\prime}(w)\right\|_{X} \leq 2\left\|f_{r}^{\prime}\right\|_{H^{\infty}(X)} \\
& =2 \sup _{|z|=r}\left\|f^{\prime}(z)\right\|_{X} .
\end{aligned}
$$

This yields that $\left\|\widetilde{V}_{n}\right\|_{\mathcal{B}(X) \rightarrow \mathcal{B}(X)} \leq 2$ for all $n$. It suffices to show that the norm $\left\|C_{\phi}-C_{\phi} \widetilde{V}_{n}\right\|$ can be made arbitrarily small by choosing $n$ large enough. Namely, the weak compactness of the boundedly supported Fourier multiplier $\widetilde{V}_{n}$ is established exactly as in the proof of Proposition 1. Condition (20) yields $r<1$ such that $\left|\phi^{\prime}(z)\right|\left(1-|z|^{2}\right) \leq \varepsilon\left(1-|\phi(z)|^{2}\right) / 3$ if $|\phi(z)| \geq r$. Proposition 1 in turn enables us to choose an integer $n$ such that for all $h \in H^{\infty}(X)$ it holds that $\left\|\left(h-V_{n} h\right)(z)\right\|_{X} \leq \varepsilon(1-r)\|h\|_{H^{\infty}(X)}$ whenever $|z| \leq \sqrt{r}$. Hence for $|z| \leq r$ we arrive at the estimate

$$
\begin{aligned}
\left\|\left(f-\widetilde{V}_{n} f\right)^{\prime}(z)\right\|_{X} & \leq \sup _{|z| \leq \sqrt{r}}\left\|\left(f_{\sqrt{r}}^{\prime}-V_{n} f_{\sqrt{r}}^{\prime}\right)(z)\right\|_{X} \\
& \leq \varepsilon(1-r)\left\|f_{\sqrt{r}}^{\prime}\right\|_{H^{\infty}(X)} \leq \varepsilon\|f\|_{\mathcal{B}(X)}
\end{aligned}
$$


according to the definition of the Bloch norm. Suppose next that $f \in \mathcal{B}(X)$ and $z \in D$ are arbitrary and consider

$$
A:=\left(1-|z|^{2}\right)\left\|\left(C_{\phi} g\right)^{\prime}(z)\right\|_{X}=\frac{\left|\phi^{\prime}(z)\left(1-|z|^{2}\right)\right|}{1-|\phi(z)|^{2}}\left\|g^{\prime}(\phi(z))\right\|_{X}\left(1-|\phi(z)|^{2}\right),
$$

where we have denoted $g:=f-\widetilde{V}_{n} f$. If $|\phi(z)|>r$, the choice of $r$ yields that $A \leq(\varepsilon / 3)\|g\|_{B(X)} \leq \varepsilon\|f\|_{B(X)}$. On the other hand, for $|\phi(z)| \leq r$ an application of the Schwarz lemma and (24) shows that $A \leq \varepsilon\|f\|_{B(X)}$. Hence $\left\|C_{\phi}-C_{\phi} \widetilde{V}_{n}\right\| \leq \varepsilon$ and the proof of part (i) is finished.

(ii) The proof of the sufficiency of the stated conditions follows from that of part (i) since (21) clearly implies (20). One only has to check that condition (21) yields that $C_{\phi}$ maps $\mathcal{B}_{0}(X)$ inside $\mathcal{B}_{0}(X)$, which is straightforward. For the necessity one notes that weak compactness of $C_{\phi}$ on $\mathcal{B}_{0}$ implies (21) according to [10, Theorems 1 and 3].

Madigan and Matheson [10, Theorem 3] observed that every weakly compact composition operator on $B_{0}$ is compact. This actually follows directly from the general fact that every weakly compact operator on $\mathcal{B}_{0}$ is compact, which is a consequence of the Gantmacher theorem [18, II.c.6.b] and the Schur property of $\mathcal{B}_{0}^{\prime}$. We record separately a special case of our Theorem 4 that complements [10, Theorem 3]. Recall that, contrary to the case of $\mathcal{B}_{0}$, there are weakly compact operators on $\mathcal{B}$ that are not compact, since $\mathcal{B} \approx \ell^{\infty}$ (see [18, Remark on p. 45]).

Corollary 5. $\quad C_{\phi}: \mathcal{B} \rightarrow \mathcal{B}$ is weakly compact only if it is compact.

In our last result about weak compactness we consider the operator $C_{\phi}$ on $H^{\infty}(X)$.

Theorem 6. $\quad C_{\phi}: H^{\infty}(X) \rightarrow H^{\infty}(X)$ is weakly compact if and only if $X$ is reflexive and

$$
\|\phi\|_{\infty}<1
$$

Proof. (i) Assume first that $X$ is reflexive and let $\phi \in H^{\infty}$ with $\|\phi\|_{\infty}<1$. We again show that $\left\|C_{\phi}-C_{\phi} V_{n}\right\|_{H^{\infty}(X) \rightarrow H^{\infty}(X)}$ can be made arbitrarily small once $n$ is large enough. Given $\varepsilon>0$, Proposition 2 yields an integer $n$ such that $\left\|f(w)-V_{n} f(w)\right\|_{X} \leq \varepsilon\|f\|_{H^{\infty}(X)}$ whenever $|w| \leq\|\phi\|_{\infty}$ and $f \in H^{\infty}(X)$. Hence we get the desired estimate

$$
\left\|\left(C_{\phi} f-C_{\phi} V_{n} f\right)(z)\right\|_{X}=\left\|\left(f-V_{n} f\right)(\phi(z))\right\|_{X} \leq \varepsilon\|f\|_{H^{\infty}(X)}
$$


for all $z \in D$. For the converse, assume that $C_{\phi}: H^{\infty}(X) \rightarrow H^{\infty}(X)$ is weakly compact. Deduce as before that $X$ is reflexive and the scalar operator $C_{\phi}: H^{\infty} \rightarrow H^{\infty}$ is weakly compact. We invoke the beautiful result, due to Ülger [17] and independently to Aron, Galindo and Lindström [1], stating that every weakly compact homomorphism on $H^{\infty}$ is compact. In our special cituation it follows that $C_{\phi}$ is compact on $H^{\infty}$, and hence that $\|\phi\|_{\infty}<1$, see [5, Exercise 3.2.2]. Alternatively, if one wants to avoid the general result for homomorphisms, a direct argument can be obtained by specializing to the case $E=\mathbf{C}$ in the proof of the implication $(2) \Rightarrow(3)$ of [1, Proposition 3].

Remark. If $\phi$ belongs to the disk algebra $A_{0}$, then the above theorem holds with the same proof also for the vector-valued 'disk algebra' $A_{0}(X)$, which is the subspace of $H^{\infty}(X)$ containing those functions that extend continuously to the closed disk $\bar{D}$.

We finally briefly consider another, somewhat related 'smallness' property of the composition operator $C_{\phi}$. Given a Banach space $E$, recall that an operator $T \in L(E)$ is weakly conditionally compact (abbreviated $T$ is w.c.c) if any bounded sequence $\left(x_{n}\right)$ in $E$ contains a subsequence $\left(x_{n_{k}}\right)$ such that the sequence $\left(T x_{n_{k}}\right)$ is weakly Cauchy. Rosenthal's $\ell^{1}$-criterion (see $[8,2 . e .5]$ ) implies that $T$ is not w.c.c if and only if $T$ fixes a copy of $\ell^{1}$ in $E$. Note that if $\ell^{1}$ does not embed into $E$, then every $T \in L(E)$ is w.c.c. Hence the set of w.c.c. operators on $E$ is, in general, strictly larger than the set of weakly compact operators on $E$. Our previous results have the following counterparts.

Theorem 7. Assume that $\ell^{1}$ does not embed in $X$. Then $C_{\phi}$ is w.c.c on $H^{1}(X)$ if and only if $\phi$ satisfies the Shapiro condition (17). Analogously, the conditions (16), (20) and (25) characterize when $C_{\phi}$ is w.c.c on $B_{1}(X)$, $\mathcal{B}(X)$ and $H^{\infty}(X)$, respectively.

Proof. The sufficiency of the stated conditions is established in each case exactly as in the proofs of Theorems 3,4 and 6 . Namely, the operators $V_{n}$ or $\widetilde{V}_{n}$ are w.c.c in the case that $\ell^{1}$ does not embed in $X$, since each summand in (7) clearly factorizes through $X$. Towards the other direction, assume that $C_{\phi}$ is w.c.c. on one of the spaces considered in the theorem. Clearly $\ell^{1}$ cannot embed in $X$ while $C_{\phi}$ fixes a copy of $X$. Moreover, since the vector-valued $C_{\phi}$ always carries a 'copy' of the corresponding scalar operator, in order to deduce the appropriate condition on $\phi$, it is enough according to Theorems 3,4 and 7.(i) to show that all w.c.c. operators on the spaces $H^{1}, B_{1}, \mathcal{B}$ and $H^{\infty}$ are weakly compact. In the cases of $H^{1}$ and $B_{1}$ this follows immediately 
from the weak sequential completeness of the spaces $L_{1}(\mu)$ (see [18, III.C.14] and recall that $H^{1}$ is a closed subspace of $L^{1}(\partial D)$ and $\left.B_{1} \approx \ell^{1}\right)$. For $H^{\infty}$ (resp. $\mathcal{B}$ ) one uses the deep result of Bourgain [3, Remark p. 45] (resp. of Rosenthal $[8,2 . f .4]$ ) stating that every non-weakly compact operator on $H^{\infty}$ (resp. $\ell^{\infty}$ ) acts isomorphically on a copy of $\ell^{\infty}$, and hence on a copy of $\ell^{1}$. Recall here that $\mathcal{B} \approx \ell^{\infty}$ (see e.g. [18, Remark on p. 45]).

\section{References}

[1] R. Aron, P. Galindo and M. Lindström, Compact homomorphisms between algebras of analytic functions, Studia Math., 123 (1997), 235-247.

[2] O. Blasco, Boundary values of functions in vector valued Hardy spaces and geometry of Banach spaces, J. Funct. Anal., 78 (1988), 346-364.

[3] J. Bourgain, New Banach space properties of the disc algebra and $H_{\infty}$, Acta Math., 152 (1984), 1-48.

[4] D. L. Burkholder, Martingales and Fourier analysis in Banach spaces, in 'Probability and Analysis', Lecture Notes in Mathematics 1206, Springer, (1986), 61-108.

[5] C.C. Cowen and B.D. MacCluer, Composition Operators on Spaces of Analytic Functions, CRC Press, Boca Raton, 1995.

[6] J. Diestel and J.J. Uhl, Vector measures, Amer. Math. Soc., Math. Surveys, 15, Providence, 1977.

[7] Y. Katznelson, An introduction to Harmonic analysis, 2nd ed., Dover, New York, 1976.

[8] J. Lindenstrauss and L. Tzafriri, Classical Banach spaces I. Sequence spaces, Ergebnisse der Mathematik, 92, Springer, 1977.

[9] B.D. MacCluer and J.H. Shapiro, Angular derivatives and composition operators on the Hardy and Bergman spaces, Canadian J. Math., 38 (1986), 878-906.

[10] K.M. Madigan and A. Matheson, Compact composition operators on the Bloch space, Trans. Amer. Math. Soc., 347 (1995), 2679-2687.

[11] J. Rubio de Francia, Martingales and integral transforms of Banach space valued functions, Lecture Notes in Mathematics 1221, Springer (1986), 195-222.

[12] D. Sarason, Weak compactness of holomorphic composition operators on $H^{1}$, in 'Functional Analysis and Operator Theory', Lecture Notes in Mathematics 1511, Springer, (1992), 75-79.

[13] J.H. Shapiro, The essential norm of a composition operator, Ann. Math., 125 (1987), 375-404.

[14] Composition operators and classical function theory, Springer, New York, 1993.

[15] J.H. Shapiro and C. Sundberg, Compact composition operators on $L^{1}$, Proc. Amer. Math. Soc., 145 (1990), 443-449.

[16] C.S. Stanton, Counting functions and majorization theorems for Jensen measures, Pacific J. Math., 125 (1986), 459-468.

[17] A. Ülger, Some results about the spectrum of commutative Banach algebras under the weak topology and applications, Monatsh. Math., 121 (1996), 353-379. 
[18] P. Wojtaszczyk, Banach spaces for analysts, Cambridge University Press, Cambridge, 1989.

[19] K. Zhu, Operator theory in function spaces, Dekker, New York, 1990.

Received December 13, 1996. The first author was partially supported by the Academy of Finland (project 34688) and the National Science Foundation of P. R. China. The second author was supported by the Academy of Finland.

WUHAN UNIVERSITY

WuHAN, Hubei 430072

P. R. CHINA

E-mail address: pdliu@whu.edu.cn

P.O. Box 4 (Yliopistonkatu 5)

FIN-00014 UNIVERSITY OF HELSINKI

FINLAND

E-mail address: saksman@cc.helsinki.fi

P.O. Box 4 (YliopistonkAtu 5)

FIN-00014 UNIVERSITY OF HELSINKI

FINLAND

E-mail address: hojtylli@cc.helsinki.fi 